\title{
Epithelial ablation of Miro1/Rhot1 GTPase augments lung inflammation by cigarette smoke
}

\author{
Shikha Sharma, Qixin Wang, Thivanka Muthumalage and Irfan Rahman*
}

Department of Environmental Medicine, University of Rochester Medical Center, Rochester, NY, USA

* Correspondence: should be addressed to: Irfan Rahman, Ph.D., Department of Environmental Medicine, University of Rochester Medical Center, Box 850, 601 Elmwood Avenue, Rochester 14642, NY, USA, Tel: 1585275 6911; E-mail: irfan_rahman@urmc.rochester.edu

Abstract: Cigarette smoke (CS) exposure results in lung damage and inflammation through mitochondrial dysfunction. Mitochondrial quality control is sustained by Miro1 (Rhot1), a calcium-binding membrane-anchored GTPase by its interaction with PINK1/Parkin during mitophagy. However, the exact mechanism that operates the interaction of Miro1 with mitophagy machinery, and their role in CS-induced mitochondrial dysfunction that results in lung inflammation remains unclear. We hypothesized that Miro1 plays an important role in regulating mitophagy machinery and resulting lung inflammation by exposure to CS in mice. We have compared the CS-induced inflammatory effects between Wild-type (Rhot $1^{\mathrm{fl} / \mathrm{fl}}$ ) and lung epithelial cell-specific Rhot1 KO mice(Rhot $\left.1^{\mathrm{CreCC} 10}\right)$ mice to determine the role of Miro1 in CSinduced inflammation and its implication on the development of lung diseases like COPD. The Rhot $1^{\mathrm{fl} / \mathrm{fl}}$ (WT) and Rhot $1^{\mathrm{CreCC} 10}$ mice were exposed to mainstream CS for 3 days (acute) and 4 months (chronic). The cellular infiltration, cytokines, and lung histopathology were studied as parameters for the inflammatory response in the lungs. Acute CS exposure showed a notable increase in the total inflammatory cells, macrophages, and neutrophils associated with inflammatory mediators. Chronic exposure showed an increase infiltration of total inflammatory cells and neutrophils versus air controls. Histopathological changes, such as macrophages and neutrophils were increased in lung interstitium of CS exposed mice associated with foamy/enlarged macrophages and epithelial hyperplasia/dysplasia. The effects of acute and chronic CS exposure were augmented in Rhot $1^{\mathrm{CreCC} 10}$ group, which indicates the epithelial Miro1 ablation led to augmentation of inflammatory cell infiltration with alteration in the levels of pro-inflammatory cytokines and histopathological changes. Overall, the findings suggest the role of Rhot1/Miro1 in regulating CS induced lung inflammatory responses with implications in mitochondrial quality control.

Keywords: Miro1; lung inflammation; mitochondrial quality control; cigarette smoke; COPD 
Short running title: Miro1 ablation augments lung inflammation

\section{Introduction}

Cigarette smoke (CS) leads to mitochondrial dysfunction associated with lung inflammation (Sundar, Maremanda, \& Rahman, 2019). Mitochondria dysfunction accelerates the inflammatory process in lung diseases like chronic obstructive pulmonary disease (COPD) (Ahmad et al., 2015). Normal cellular homeostasis and physiology depend on the regulation of mitochondrial function (Ni, Williams, \& Ding, 2015). During cellular damages, dysfunctional mitochondria are eliminated by a selective degradation process, mitophagy (Lazarou et al., 2015). To sustain the quality control of the mitochondria, Miro1 (Rhot1) serves as an essential element in mitochondrial quality control. Rhot1 (Miro1) is a calcium-binding membrane-anchored GTPase that is required for the calcium-driven movement of the mitochondria on microtubules, especially during mitophagy (Alshaabi et al., 2021; Sundar et al., 2019; Cloonan \& Choi, 2016; Lerner et al., 2016). Miro1 also serves a vital role in mitochondrial dynamics by its interaction with Pink1 (PTEN-induced putative kinase 1)/Parkin mitochondrial quality control system by serving as a signal for mitophagy (Bueno et al., 2015; Safiulina et al., 2019). Depolarization of the mitochondrial membrane is responsible for initiating mitophagy in the cells. Following this, the mitochondrial quality control is performed by the stabilization of Pink1 on mitochondrial outer membranes through fission reaction. An E3 ubiquitin ligase, also called Parkin, is recruited from the cytosol by the Pink1 for further mitochondrial quality control (Scarffe, Stevens, Dawson, \& Dawson, 2014; Sundar et al., 2019). After the recruitment of Parkin, the degradation of a mitochondrial fusion core protein - mitofusin2 (Mfn2) occurs. This allows clearing the damaged mitochondria from the cell by protein microtubuleassociated protein light chain 3 (LC3) in the isolation membranes. This phenomenon further initiates the formation of autophagosome by localization of damaged mitochondria and that in turn fuses with the lysosomes to remove the dead mitochondrial cells (Ashrafi \& Schwarz, 2013; Sekine \& Youle, 2018). The Pink1 and Parkin thereby coordinate together during mitophagy to help regulate mitochondrial degradation. The mitochondrial shape and trafficking are maintained by GTPase Miro1 and Miro2. Thus, together they may serve a crucial role in mitochondrial quality control (Nemani et al., 2018). However, the exact mechanism that operates this interaction and the role of Miro1 in CS-induced mitochondrial dysfunction resulting in lung inflammation in COPD remains unclear.

We hypothesize that the ablation of Miro1 (Rhot1) in lung epithelial cells would result in the dysfunction of mitochondrial quality control (dysfunctional mitophagy). This may in turn exaggerate the adverse effects of cigarette smoke in the lungs, thereby ensuing CS-induced lung inflammation. (Lerner, Sundar, et al., 2016; Sundar et al., 2019). To determine the role of Rhot1/Miro1 in augmenting the CS-induced lung inflammation and its implication on COPD emphysema phenotype development, we utilized the epithelial cell-specific (Rhot1 ${ }^{\mathrm{CreCC} 10}$ ) Miro1 knockout mouse models which are to exposed to mainstream CS for 3 days (acute phase), and 4 months (chronic phase). The role of mitochondrial Miro1 and its impact on 
the progression of lung inflammation by acute and chronic CS exposures to mice was determined.

\section{Materials and Methods}

Ethical Approval: Institutional Biosafety

\section{Ethics Statement}

The study is approved via the laboratory protocols by the Institutional Biosafety Committee (IBC) of the University of Rochester Medical Center, Rochester, NY. All animal experiments were approved by the University Committee on Animal Research at the University of Rochester, Protocol no.102204 / 2007-070E, date of approval, January 31, 2019.

\subsection{Animal Model and exposure}

Wild-type (Rhot $1^{\mathrm{fl} / \mathrm{fl}}$ ) and lung epithelial cell-specific Rhot1 KO mice (Rhot1 ${ }^{\mathrm{CreCC} 10}$ ) models were exposed to CS for 3 days duration (acute Rhot1flp CreCC10)) and 4 months (chronic - Rhot1Flox CreCC10) (Both flp and flox techniques are based on the LoxP/Cre-mediated conditional gene $\mathrm{KO}$ and are widely used for studying gene specific functions), using Baumgartner-Jaeger CSM2082i (cigarette smoke generating machine; $\mathrm{CH}$ Technologies, Westwood, NJ, USA) (J. Chen et al., 2019; Sundar et al., 2010; Yang et al., 2021). All the air group mice were exposed to filtered room air and the same time duration was maintained as for the CS exposure. Hence, the mice groups were divided into two groups - air and CS groups. Animals were 2-4 months old and were housed under standard pathogen-free conditions with a 12/12 $\mathrm{h}$ light and dark cycle in the University of Rochester Medical Center vivarium facility.

\subsection{Cigarette smoke exposure}

Cigarette smoke for exposure was generated by using 3R4F (researchgrade cigarettes) and the exposure guidelines were followed as per the protocol of the Federal Trade Commission (1 puff/minute of 2-second duration with the volume of $35 \mathrm{ml}$ ). The mainstream smoke concentration was set at $250-300 \mathrm{mg} / \mathrm{m}^{3}$ of TPM value after diluting it with filtered air and the concentration of carbon monoxide (290 to $300 \mathrm{ppm}$ ) was monitored in the chamber (Arunachalam, Sundar, Hwang, Yao, \& Rahman, 2010; Hwang et al., 2011; Lerner, Lei, Sundar, \& Rahman, 2016). A MicroDust Pro-aerosol monitor (Casella CEL, Bedford, UK) was used for CS exposure monitoring and the verification of the readings was performed after the completion of the exposure cycle each day by gravimetric sampling. Simultaneously, all the air group mice were exposed to the same time duration of normal air as maintained for the CS exposure (Sundar et al., 2010; Yao et al., 2008).

\subsection{Collection of bronchoalveolar lavage (BAL)}

Mice were injected with $100 \mathrm{mg} / \mathrm{kg}$ (BW) of pentobarbital sodium (Abbott Laborotaries, Abbott Park, IL) intraperitoneally and then euthanized. Then cannula was inserted into the trachea of mice and the lungs were lavaged with normal saline ( $0.6 \mathrm{ml}$ volume; 3 times). The lavage fluid was collected and centrifuged and the separated supernatant samples were stored 
at $-80^{\circ} \mathrm{C}$ (M. Chen et al., 2017; Rajendrasozhan, Chung, Sundar, Yao, \& Rahman, 2010; Wood et al., 2014; Yao et al., 2008).

\subsection{Total cell count in BAL fluid}

The cell pellet of BAL fluid was resuspended in normal saline $(0.9 \%$ $\mathrm{NaCl} ; 1 \mathrm{ml} / \mathrm{vol}$.) and stained by trypan blue (Cat\# 15250061, Thermo Fisher Scientific) and total cell count $/ \mathrm{ml}$ was determined by TC-20 Automated Cell Counter (BioRad).

\subsection{Differential cell count in BAL fluid}

For 3 days (acute) exposure, the differential cell count of immuneinflammatory cells (neutrophils, macrophages, CD4 T-lymphocytes, CD8 Tlymphocytes) was done by Guava ${ }^{\circledR}$ easyCyte ${ }^{\mathrm{TM}}$ flow cytometer (Millipore Sigma) in BAL fluid. All cells were stained using cell type-specific mAb. Specific cell labeling markers, LY6B.2 Alexa Fluor 488 (Cat\#NBP213077AF488, Novus Biologicals), F4/80 phycoerythrin (Cat\#123109, BioLegend), CD45 allophycocyanin (Cat\#110728, BioLegend), CD4a PE-Cy7 (Cat\#25-0041-82, Fisher Scientific) and CD8a phycoerythrinCy5 (Cat\#17-0081-82, Fisher Scientific)) for neutrophils, macrophages, leukocytes, and T-lymphocytes were used, respectively.

For four months (chronic) exposure, differential cell counts to check the cell influx in neutrophils and macrophages were performed on cytospin slides with Diff-Quick staining (Cui, Liu, Ip, Liang, \& Mak, 2020; Lim, Kim, Lee, Bae, \& Kim, 2018). The Diff-Quick staining involves sequential dipping of the slides into different solutions - fixative agent (methanol, blue), solution 1 (eosinophilic, orange), and solution 2 (basophilic, blue) followed by rinsing and drying. The collected smears were first allowed to dry followed by dipping the slide for one second and repeating it for five times each into a fixative followed by stains 1 and 2 . The excess was allowed to drain after each dip. After drying it, the slide was rinsed in distilled water or Weise's buffer ( $\mathrm{pH}$ 7.2). It is then blotted or allowed to dry in the air for further examination at low magnification and under oil immersion.

\subsection{Inflammatory mediators}

Differential levels of inflammatory mediators in BAL fluid were assessed for their alteration in the levels using Bio-Plex Pro Mouse Cytokine 23-Plex Immunoassay (Cat\# M60009RDPD, BioRad.) was run using Luminex. Experiments were done following the manufacturer's instructions and the results were presented as $\mathrm{pg} / \mathrm{ml}$ in the samples.

\subsection{Measurement of lung mechanics}

The mechanical parameters of the lungs, such as lung resistance, tissue elastance, and static compliance were measured by Flexivent apparatus (Scireq; Montreal, Canada). All the mice were weighed and anesthetized by pentobarbital $(90 \mathrm{mg} / \mathrm{kg}$; i.p. injection). Mice were then tracheostomized and cannulated and the cannula was attached to the rodent ventilator and the ventilator was connected to a computer (Hwang et al., 2011; Phillips et al., 2015). Measurements of all lung mechanical parameters were repeated three times for all mice. 


\subsection{Lung Morphometry and histopathology}

Mouse lungs were isolated and inflated with 1\% agarose (low melting) and fixed with $4 \%$ neutral-buffered paraformaldehyde (Yao et al., 2010). Dehydration of the fixed tissue was done followed by encasing it in paraffin and sections were cut by rotary microtome. The midsagittal lung section of each tissue was stained with H\&E (hematoxylin and eosin) and the Lm (linear intercept) of airspace and histopathological changes were determined in lung tissues (20X) using Metamorph software.

\subsection{Statistical Analysis}

Statistical analysis was done by one-way (ANOVA), Tuckey's post-hock multiple group comparison test by GraphPad Prism 9 software. Data were shown as mean \pm SEM. Significance compared between corresponding Air and CS groups of same genotypes as well as in different genotypes. $P<0.05$ is considered significant.

\section{Results}

CS-induced inflammatory cell infiltration and differential expression of cytokines were observed in lung epithelial cell-specific Rhot1 deleted mice after 3 days acute and 4 months chronic of CS exposure.

\subsection{Three days (acute) exposure}

Inflammatory cellular influx in Rhot1 epithelial cell specific KO $\left(\right.$ Rhot $\left.1^{\mathrm{CreCC} 10}\right)$ and WT (Rhot $\left.1^{\mathrm{fl} / \mathrm{fl}}\right)$ mice.

The total number of cells, macrophage and neutrophil counts were increased significantly only in Rhot1 epithelial cell specific KO (Rhot1Crecc10) mice exposed to CS for 3 days, whereas the upregulation trends were observed in WT (Rhot1 $1^{\mathrm{f} / \mathrm{fl}}$ ) mice exposed to CS without significance. However, none of the groups showed significant changes in the levels of CD4 and CD8 T-lymphocytes in response to CS exposure (Figure 1).

3.1.1. Effect of acute, CS exposure on levels of pro-inflammatory mediators in Rhot1 epithelial cell specific KO (Rhot1 $\left.{ }^{\mathrm{CreCC10}}\right)$ and WT (Rhot1 $\left.{ }^{\mathrm{fl} / \mathrm{fl}}\right)$ mice

WT (Rhot $1^{\mathrm{f} / \mathrm{fl}}$ ) mice exposed to 3 days CS showed increasing trends of several pro-inflammatory mediators, but the changes between the air and CS group were not significant. CS exposed Rhot1 epithelial cell specific KO (Rhot1 ${ }^{\mathrm{CreCC} 10}$ ) mice showed a significant reduction in the levels of MCP1, IL5, Il-1a, IL-3, IL-9, IFN- $\gamma$, and TNF- $\alpha$. Interestingly, a significant reduction in the levels of IL17a, IL4 and KC were observed in WT (Rhot1 ${ }^{\mathrm{fl} / \mathrm{fl}}$ ) CS groups. The rest of the analyzed mediators were not significantly affected (Figure 2).

\subsection{Four Months (chronic) exposure}

3.2.1. Inflammatory cellular influx in Rhot1 epithelial cell specific KO (Rhot1 +-CreCC10 and Rhot1 ${ }^{--C}$ reCC10) and WT (Rhot1 Flox) mice by chronic CS exposures

Chronic CS exposure significantly increased the total cells and number of neutrophils in Rhot ${ }^{\mathrm{CreCC} 10}$ mice. Non-significant, but increasing trend was observed in number of macrophages in CS exposed groups (Figure 3). 
3.2.2. Effect of chronic, CS exposure on levels of pro-inflammatory mediators in Rhot1 epithelial cell specific KO (Rhot1 $\left.\left.{ }^{\mathrm{CreCC} 10}\right)\right)$ and WT $\left.\left(\operatorname{Rhot}^{\mathrm{fl} / \mathrm{f}}\right)\right)$ mice.

CS exposed WT $\left(\operatorname{Rhot}^{\mathrm{fl} / \mathrm{fl}}\right)$ and Rhot1 epithelial cell specific KO (Rhot1 ${ }^{\mathrm{CreCC} 10}$ ) mice showed significant increased levels cytokines (MCP-1, KC, IL-12p40, and MIP-1 $\alpha$.). Intriguingly, the levels of MCP-1, MIP- $1 \alpha$, and MIP-1 $\beta$ in BALF were augmented in Rhot1 epithelial cell specific KO $\left(\right.$ Rhot $1^{\mathrm{CreCC} 10}$ ) mice than WT (Rhot $1^{\mathrm{fl} / \mathrm{fl}}$ ) mice. However, the levels of IL-13, IL10 , MIP1- $\beta$, G-CSF, and IL-12p70 showed a significant increase only in Rhot1 epithelial cell specific $\mathrm{KO}\left(\operatorname{Rhot} 1^{\mathrm{CreC} C 10}\right)$ mice. The rest of the mediators were not affected significantly (Figure 4).

3.2.3. Effect of chronic CS on lung mechanical properties in Rhot1 epithelial cell specific KO (Rhot1 $\left.{ }^{\mathrm{CreCC} 10}\right) \mathrm{WT}\left(\right.$ Rhot $\left.^{\mathrm{fl} / \mathrm{fl}}\right)$ mice

WT (Rhot1 ${ }^{\mathrm{fl} / \mathrm{fl})}$ and KO (Rhot1 $\left.{ }^{\mathrm{CreCC} 10}\right)$ mice exposed to CS showed no significant changes in parameters of lung mechanical properties, such as resistance, compliance, and elastance (Figure 5).

3.2.4. Effect on airspace enlargement and histopathology of lung tissues in Rhot1 epithelial cell specific KO (Rhot $\left.1^{\mathrm{CreCC} 10}\right)$ and WT (Rhot1 $\left.{ }^{\mathrm{f} / \mathrm{fl} 1}\right)$ mice

Lung sections from chronic 4 months CS-exposed both WT (Rhot1 $1^{\mathrm{f} / \mathrm{f}}$ ) and $\mathrm{KO}\left(\right.$ Rhot $\left.1^{\mathrm{CreCC10}}\right)$ mice did not show significant airspace enlargement in comparison with air group. Differences in the age and sex of the animals might have caused the differences observed in the baseline. However, other histopathological changes, such as interstitial foamy macrophages and neutrophils were found in CS exposed mice and the changes were more intensified in Rhot $1{ }^{\mathrm{CreCC} 10}$ mice (Figure 6). This was associated with epithelial hyperplasia and dysplasia (columnar cell-dysplasia), implicating the role of epithelial Miro1 in regulating epithelium integrity, and inflammatory cell influx is directly associated with epithelial damage.

\section{Discussion}

Chronic inflammation of the lungs is the hallmark of the pathogenesis of COPD, which is primarily caused by cigarette smoking. Cigarette smoke contains large amounts of toxic chemicals that affect the cells of the lungs by the deleterious nature of free radicals (Nyunoya et al., 2014; Yao et al., 2012). The dysfunction in mitochondria due to injury and morphological alterations are shown to be significantly driven by CS exposure to the lung cells that includes airway and alveolar epithelial cells, fibroblasts, and airway smooth muscle cells (Aravamudan et al., 2014; Ballweg, Mutze, Konigshoff, Eickelberg, \& Meiners, 2014; Hara et al., 2013). However, the mechanism behind this cellular damage and inflammatory response, and the role of Miro1 in mitochondrial dysfunction upon exposure to CS in lung epithelial cells has remained unclear. We have previously reported the association of Miro1 reduction with CS induced inflammation in lung epithelial cells (Sundar et al.,2019). Therefore, based on our findings and reported studies on the significant role of Miro1 in regulating the mitochondrial quality control system, we embarked to study the severity of CS induced lung inflammation 
in the absence of Miro1 in epithelial cells and its association with mitochondrial dysfunction.

In this study, the Rhot $1^{\mathrm{fl} / \mathrm{fl}}$ (WT) and lung epithelial cell-specific Rhot1 ${ }^{\mathrm{CreCC} 10} \mathrm{KO}$ mice were exposed to CS on acute (3 days) and chronic duration (4 months) and the comparative study of inflammatory responses between Rhot1 $1^{\mathrm{fl} / \mathrm{fl}}$ (WT) and Rhot1 ${ }^{\mathrm{CreCC} 10}(\mathrm{KO})$ animals was performed to determine the role of Rhot1/Miro1 in the CS-induced lung inflammation and epithelial integrity. The role of mitochondrial dysfunction in lung inflammatory diseases, such as COPD is known (Mizumura et al., 2014; Wiegman et al., 2015). Regulation of cellular events like fission (fragmentation) and fusion (elongation) process allows mitochondria to rapidly change their shape. Mitofusins (Mfns, membrane-anchored proteins: Mfn1 and 2) and OPA-1 (optic atrophy 1) are the major proteins that allow mitochondrial fusion by interacting with other proteins like Pink1 in the mitochondrial membranes (Y. Chen \& Dorn, 2013). The association of Miro1 mediated mitochondrial dysregulation in the augmentation of inflammatory responses was evident in our study as a result of intensified increase in the lung infiltration of total inflammatory cells, macrophages, and neutrophils in bronchoalveolar lavage (BAL) fluid in $\operatorname{Rhot} 1^{\mathrm{CreCC} 10}(\mathrm{KO})$ mice after acute and chronic exposures of CS. However, there was no change observed in the CD4 and CD8 cell counts. A significant increase in macrophages and neutrophils suggests association of inflammatory cell influx with the acute and chronic lung inflammation. However, the significant reduction and decreasing trends in inflammatory mediators after 3 days acute exposure, such as MCP1, IL5, IL-1a, IL-3, IL-9, IFN- $\gamma$, KC and TNF- $\alpha$ in Miro1 CreCC10 KO group, after the acute CS exposure points towards the possibility that the inflammatory cells may have undergone morphological changes which have reduced the release of these mediators. Moreover, the exaggerated abundances in MCP-1, MIP$1 \beta$ and MIP1- $\alpha$ after chronic CS exposure in $\operatorname{Rhot} 1^{\mathrm{CreCC} 10}(\mathrm{KO})$ group indicate the subsequent occurrences of cell infiltration and the association of Miro1 in inducing and intensifying these changes. Further, it substantiates that any prolonged accumulation of infiltrating cells, such as neutrophils and macrophages act as prime events in the pathological contribution of cigarette/tobacco exposure to lung (Domínguez-Fandos et al., 2012; Gorska et al., 2008).

The disruptions in the mitochondria refer to various events that occur at the cellular level in the mitochondria, such as morphological changes, alteration in its metabolic activity, decreased membrane potential and altered mitochondrial superoxide levels and intracellular $\mathrm{Ca}^{2+}$ flux (Cloonan \& Choi, 2016; Lerner, Sundar, et al., 2016). This may alter the dynamic nature of the organelle. CS exposure causes an alteration in this dysregulation of mitochondria by modifying its function, mitophagy and resulting in ROS production (Ahmad et al., 2015; Hara et al., 2013). The histopathological data of the lung sections after chronic CS-exposure in Rhot1 ${ }^{\mathrm{CreCC} 10}(\mathrm{KO})$ mice did not reveal any significant airspace enlargement in comparison with air and CS-exposed Rhot1 ${ }^{\mathrm{fl} / \mathrm{fl}}$ (WT) mice. Nevertheless, other changes, such as accumulation of pulmonary interstitial macrophages and signs of epithelial hyperplasia/dysplasia (columnar cell-dysplasia) at airway region of the CS exposed mouse lungs were observed. We observed the presence of foamy macrophages in lung interstitium (macrophages undergone structural 
changes) after the 4 months CS exposure in lung tissues. The changes were more intensified in Rhot $1{ }^{\mathrm{CreCC} 10}(\mathrm{KO})$. Interestingly, G-CSF and KC which regulate the release and molecular characterization of granulocytes, found increased in Rhot1 ${ }^{\mathrm{CrCC} 10}$ (KO ) mice after 4 months of chronic CS exposure which also supports the occurrence of epithelial hyperplasia/dysplasia, and lung inflammatory cell influx is directly associated with epithelial injurious responses. The inflammation of the lungs impacts the airways with smaller airways being more predominant at the early stages of COPD (DomínguezFandos et al., 2012; Lerner, Sundar, et al., 2016). Mechanical properties and airspace enlargement of lungs had no comparable changes of Rhot1 to air and CS groups as well as to WT and Rhot1 ${ }^{\mathrm{CreCC} 10}(\mathrm{KO})$ groups. Relative shorter duration and amount of CS exposure may explain the non-significance of structural changes in these mice. There are several studies that point the role of Miro1/Rhot1 in mitochondrial quality control mechanism (Ballweg et al., 2014; Klosowiak et al., 2016; Kondapalli et al., 2012; Lopez-Domenech et al., 2018; Safiulina et al., 2019). Our findings strengthen the concept that Miro1 is a key player in exerting the inflammatory responses due to CS exposure in lungs, which in part mediated by CS-induced mitochondrial dysfunction/mitophagy and mitochondrial quality control. The mechanism identified here for the role of Miro1 may provide insights into the strategies that focus to pharmacologically restoring the role of mitochondrial quality control mechanisms to treat lung inflammatory conditions, such as COPD.

\section{Conclusion}

Epithelial Rhot/Miro1 ablation was shown to augment the inflammatory responses in lungs associated with cell infiltration with alteration in the levels of pro-inflammatory cytokines and histopathological changes in mouse lungs (Figure 7). Our findings provide insights for further studies to determine the role of Miro1 in regulating the mitochondrial quality control mechanisms by CS in the pathogenesis of COPD/emphysema. This may have implications in developing strategies for pharmacological manipulation of Miro1/mitochondrial quality control mechanisms in therapeutic strategies for lung diseases.

Acknowledgments: The National Institutes of Health (NIH) 1R01HL135613, R01 ES029177, HL137738, and R01 HL133404 supported this study. The funding body has no role in design of the study, data collection, analysis, and interpretation of data and in writing the manuscript. We thank Dr. Isaac Sundar for providing scientific/technical inputs. Dr. Krishna Maremanda provided acute exposure and cell counts as a part of technical assistance, and Dr. Lakshmi Chakrapani for technical assistance. We also thank our technical staff Daria M Krenitsky for helping us with tissue sectioning and $\mathrm{H} \& \mathrm{E}$ staining.

Author Contributions: SS designed and conducted the experiments; IR conceived the concept and ideas; TM Performed exposure design, mouse experiments, flow data analysis, and scientific input; QW Performed mouse sacrifice and exposures, histology, Lm, and data analysis; TM edited the manuscript; SS and IR wrote and/or edited/revised the manuscript. IR obtained research funding.

Declarations and Disclosures: Conflict of or competing interest statement. The authors have declared that no competing interests exist. 


\section{References}

Ahmad, T., Sundar, I. K., Lerner, C. A., Gerloff, J., Tormos, A. M., Yao, H., \& Rahman, I. (2015). Impaired mitophagy leads to cigarette smoke stress induced cellular senescence: implications for chronic obstructive pulmonary disease. The FASEB Journal, 29(7), 2912-2929.

Alshaabi, H., Shannon, N., Gravelle, R., Milczarek, S., Messier, T., \& Cunniff, B. (2021). Miro1-mediated mitochondrial positioning supports subcellular redox status. Redox Biology, 38, 101818.

Aravamudan, B., Kiel, A., Freeman, M., Delmotte, P., Thompson, M., Vassallo, R., . Prakash, Y. S. (2014). Cigarette smoke-induced mitochondrial fragmentation and dysfunction in human airway smooth muscle. American Journal of Physiology-Lung Cellular and Molecular Physiology, 306(9), L840-L854.

Arunachalam, G., Sundar, I. K., Hwang, J., Yao, H., \& Rahman, I. (2010). Emphysema is associated with increased inflammation in lungs of atherosclerosis-prone mice by cigarette smoke: implications in comorbidities of COPD. Journal of Inflammation, 7(1), 1-10.

Ashrafi, G., \& Schwarz, T. L. (2013). The pathways of mitophagy for quality control and clearance of mitochondria. Cell Death \& Differentiation,

Ballweg, K., Mutze, K., Konigshoff, M., Eickelberg, O., \& Meiners, S. (2014). Cigarette smoke extract affects mitochondrial function in alveolar epithelial cells. American Journal of Physiology-Lung Cellular and Molecular Physiology, 307(11), L895-L907.

Bueno, M., Lai, Y.-C., Romero, Y., Brands, J., Croix, C. M. S., Kamga, C., . Lee, J. S. (2015). PINK1 deficiency impairs mitochondrial homeostasis and promotes lung fibrosis. The Journal of Clinical Investigation, 125(2), 521-538.

Chen, J., Dai, L., Wang, T., He, J., Wang, Y., \& Wen, F. (2019). The elevated CXCL5 levels in circulation are associated with lung function decline in COPD patients and cigarette smoking-induced mouse model of COPD. Annals of Medicine, 51(5-6), 314-329.

Chen, M., Wang, T., Shen, Y., Xu, D., Li, X., An, J., . Chen, L. (2017). Knockout of RAGE ameliorates mainstream cigarette smoke-induced airway inflammation in mice. International Immunopharmacology, 50, 230-235.

Chen, Y., \& Dorn, G. W. (2013). PINK1-phosphorylated mitofusin 2 is a Parkin receptor for culling damaged mitochondria. Science, 340(6131), 471-475.

Cloonan, S. M., \& Choi, A. M. K. (2016). Mitochondria in lung disease. The Journal of Clinical Investigation, 126(3), 809-820.

Cui, Y., Liu, K. W. K., Ip, M. S. M., Liang, Y., \& Mak, J. C. W. (2020). Protective effect of selegiline on cigarette smokeinduced oxidative stress and inflammation in rat lungs in vivo. Annals of Translational Medicine, 8(21).

Domínguez-Fandos, D., Peinado, V. I., Puig-Pey, R., Ferrer, E., Musri, M. M., Ramírez, J., \& Barberà, J. A. (2012). Pulmonary Inflammatory Reaction and Structural Changes Induced by Cigarette Smoke Exposure in the Guinea Pig. COPD: Journal of Chronic Obstructive Pulmonary Disease, 9(5), 473-484.

Gorska, K., Krenke, R., Korczynski, P., Kosciuch, J., Domagala-Kulawik, J., \& Chazan, R. (2008). Eosinophilic airway inflammation in chronic obstructive pulmonary disease and asthma. J Physiol Pharmacol, 59(Suppl 6), 261-270.

Hara, H., Araya, J., Ito, S., Kobayashi, K., Takasaka, N., Yoshii, Y., . Numata, T. (2013). Mitochondrial fragmentation in cigarette smoke-induced bronchial epithelial cell senescence. American Journal of Physiology-Lung Cellular and Molecular Physiology, 305(10), L737-L746.

Hwang, J., Rajendrasozhan, S., Yao, H., Chung, S., Sundar, I. K., Huyck, H. L., . Rahman, I. (2011). FOXO3 deficiency leads to increased susceptibility to cigarette smoke-induced inflammation, airspace enlargement, and chronic obstructive pulmonary disease. The Journal of Immunology, 187(2), 987-998.

Ito, S., Araya, J., Kurita, Y., Kobayashi, K., Takasaka, N., Yoshida, M., . Fujii, S. (2015). PARK2-mediated mitophagy is involved in regulation of HBEC senescence in COPD pathogenesis. Autophagy, 11(3), 547-559.

Klosowiak, J. L., Park, S., Smith, K. P., French, M. E., Focia, P. J., Freymann, D. M., \& Rice, S. E. (2016). Structural insights into Parkin substrate lysine targeting from minimal Miro substrates. Scientific Reports, 6(1), 1-13.

Kobayashi, K., Araya, J., Minagawa, S., Hara, H., Saito, N., Kadota, T., . Kurita, Y. (2016). Involvement of PARK2mediated mitophagy in idiopathic pulmonary fibrosis pathogenesis. The Journal of Immunology, 197(2), 504-516.

Kondapalli, C., Kazlauskaite, A., Zhang, N., Woodroof, H. I., Campbell, D. G., Gourlay, R., . Deak, M. (2012). PINK1 is activated by mitochondrial membrane potential depolarization and stimulates Parkin E3 ligase activity by phosphorylating Serine 65. Open Biology, 2(5), 120080.

Lazarou, M., Sliter, D. A., Kane, L. A., Sarraf, S. A., Wang, C., Burman, J. L., . Youle, R. J. (2015). The ubiquitin kinase PINK1 recruits autophagy receptors to induce mitophagy. Nature, 524(7565), 309-314.

Lerner, C. A., Lei, W., Sundar, I. K., \& Rahman, I. (2016). Genetic ablation of CXCR2 protects against cigarette smokeinduced lung inflammation and injury. Frontiers in Pharmacology, 7, 391. 
Lerner, C. A., Sundar, I. K., \& Rahman, I. (2016). Mitochondrial redox system, dynamics, and dysfunction in lung inflammaging and COPD. The International Journal of Biochemistry \& Cell Biology, 81, 294-306.

Lim, D., Kim, W., Lee, C., Bae, H., \& Kim, J. (2018). Macrophage depletion protects against cigarette smoke-induced inflammatory response in the mouse colon and lung. Frontiers in Physiology, 9, 47.

Lopez-Domenech, G., Covill-Cooke, C., Howden, J. H., Birsa, N., Morfill, C., Brandon, N. J., \& Kittler, J. T. (2018). Miro ubiquitination is critical for efficient damage-induced PINK1/Parkin-mediated mitophagy. BioRxiv,

Mizumura, K., Cloonan, S. M., Nakahira, K., Bhashyam, A. R., Cervo, M., Kitada, T., . Washko, G. R. (2014). Mitophagydependent necroptosis contributes to the pathogenesis of COPD. The Journal of Clinical Investigation, 124(9), 3987-4003.

Nemani, N., Carvalho, E., Tomar, D., Dong, Z., Ketschek, A., Breves, S. L., . Palaniappan, P. (2018). MIRO-1 determines mitochondrial shape transition upon GPCR activation and Ca2+ stress. Cell Reports, 23(4), 1005-1019.

Ni, H.-M., Williams, J. A., \& Ding, W.-X. (2015). Mitochondrial dynamics and mitochondrial quality control. Redox Biology, 4, 6-13.

Nyunoya, T., Mebratu, Y., Contreras, A., Delgado, M., Chand, H. S., \& Tesfaigzi, Y. (2014). Molecular processes that drive cigarette smoke-induced epithelial cell fate of the lung. American Journal of Respiratory Cell and Molecular Biology, 50(3), 471-482.

Phillips, B., Veljkovic, E., Peck, M. J., Buettner, A., Elamin, A., Guedj, E., . Bou, S. (2015). A 7-month cigarette smoke inhalation study in C57BL/6 mice demonstrates reduced lung inflammation and emphysema following smoking cessation or aerosol exposure from a prototypic modified risk tobacco product. Food and Chemical Toxicology,

80, 328-345.

Rajendrasozhan, S., Chung, S., Sundar, I. K., Yao, H., \& Rahman, I. (2010). Targeted disruption of NF- B1 (p50) augments cigarette smoke-induced lung inflammation and emphysema in mice: a critical role of p50 in chromatin remodeling. American Journal of Physiology-Lung Cellular and Molecular Physiology, 298(2),

Safiulina, D., Kuum, M., Choubey, V., Gogichaishvili, N., Liiv, J., Hickey, M. A., . Liiv, M. (2019). Miro proteins prime mitochondria for Parkin translocation and mitophagy. The EMBO Journal, 38(2), e99384.

Scarffe, L. A., Stevens, D. A., Dawson, V. L., \& Dawson, T. M. (2014). Parkin and PINK1: much more than mitophagy. Trends in Neurosciences, 37(6), 315-324.

Sekine, S., \& Youle, R. J. (2018). PINK1 import regulation; a fine system to convey mitochondrial stress to the cytosol. BMC Biology, 16(1), 1-12.

Sundar, I. K., Chung, S., Hwang, J.-W., Arunachalam, G., Cook, S., Yao, H., . Rahman, I. (2010). Peroxiredoxin 6 differentially regulates acute and chronic cigarette smoke mediated lung inflammatory response and injury. Experimental Lung Research, 36(8), 451-462.

Sundar, I. K., Maremanda, K. P., \& Rahman, I. (2019). Mitochondrial dysfunction is associated with Miro1 reduction in lung epithelial cells by cigarette smoke. Toxicology Letters, 317, 92-101.

Wiegman, C. H., Michaeloudes, C., Haji, G., Narang, P., Clarke, C. J., Russell, K. E., . Kanerva, J. (2015). Oxidative stressinduced mitochondrial dysfunction drives inflammation and airway smooth muscle remodeling in patients with chronic obstructive pulmonary disease. Journal of Allergy and Clinical Immunology, 136(3), 769-780.

Wood, T. T., Winden, D. R., Marlor, D. R., Wright, A. J., Jones, C. M., Chavarria, M., . Reynolds, P. R. (2014). Acute secondhand smoke-induced pulmonary inflammation is diminished in RAGE knockout mice. American Journal of Physiology-Lung Cellular and Molecular Physiology, 307(10), L758-L764.

Yang, D., Xu, D., Wang, T., Yuan, Z., Liu, L., Shen, Y., \& Wen, F. (2021). Mitoquinone ameliorates cigarette smokeinduced airway inflammation and mucus hypersecretion in mice. International Immunopharmacology, 90, 107149.

Yao, H., Chung, S., Hwang, J., Rajendrasozhan, S., Sundar, I. K., Dean, D. A., . Rnty, M. (2012). SIRT1 protects against emphysema via FOXO3-mediated reduction of premature senescence in mice. The Journal of Clinical Investigation, 122(6), 2032-2045.

Yao, H., Edirisinghe, I., Rajendrasozhan, S., Yang, S.-R., Caito, S., Adenuga, D., \& Rahman, I. (2008). Cigarette smokemediated inflammatory and oxidative responses are strain-dependent in mice. American Journal of PhysiologyLung Cellular and Molecular Physiology, 294(6),L1174-L1186. Retrieved from https://journals.physiology.org/doi/abs/10.1152/ajplung.00439.2007 


\section{Figures and Figure Legends}

Figure 1

A

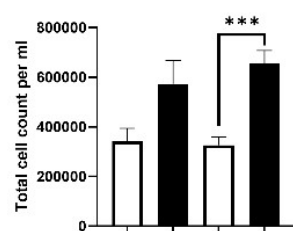

D

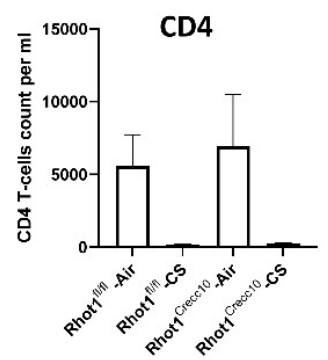

B
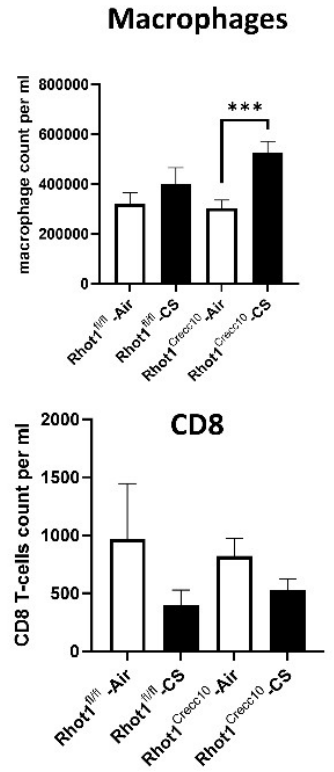

C

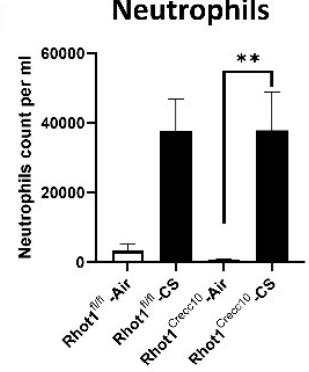

Figure 1: CS induced cell infiltration in lung epithelial cell-specific Rhot1 deleted mice: Rhot $1^{\mathrm{fl} / \mathrm{fl}}$ (WT) and Rhot $1^{\mathrm{CreCC} 10}$ (Rhot1 flp $\mathrm{CreCC} 10^{+/-}$and Rhot1 flp $\mathrm{CreCC} 10^{+/+}$) mice were exposed to room air and CS (mainstream) for 3 days (acute exposure). Differential cell count in BAL fluid filtered air and CS-exposed mice for 3 days was determined. Alteration in A. Total cell count, B. Macrophage, C. Neutrophils, D. CD4 T-cells and E. CD8 T-cells, occurred due to CS exposure. Significance compared between corresponding Air and CS groups and all the groups compared with each other irrespective of their exposures. Data are shown as mean \pm SEM ( $\mathrm{n}=5$ to 13 per group). $* * P<0.01, * * * P<0.001$ 
Figure 2
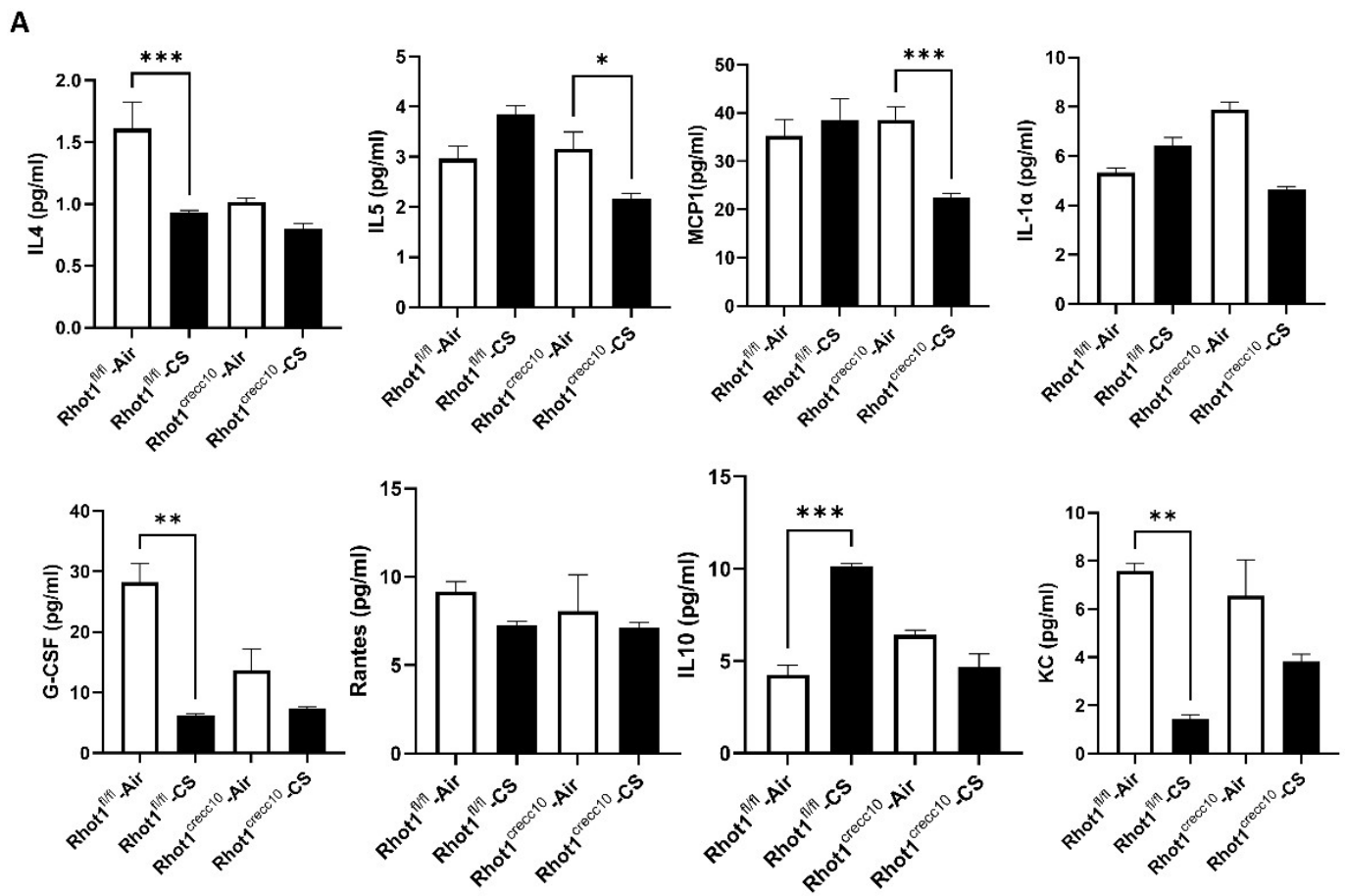
Figure 2

B
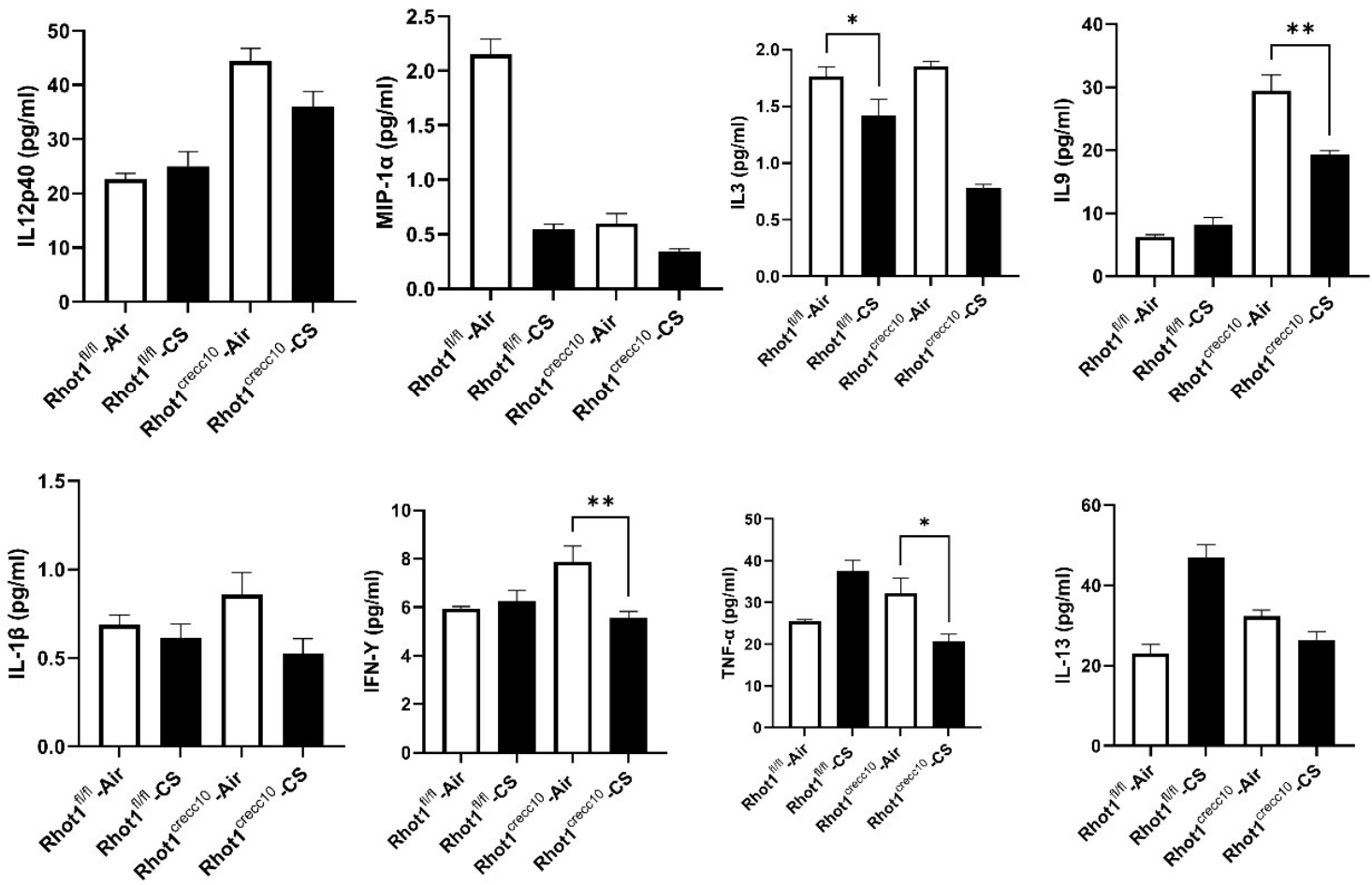
Figure 2

C
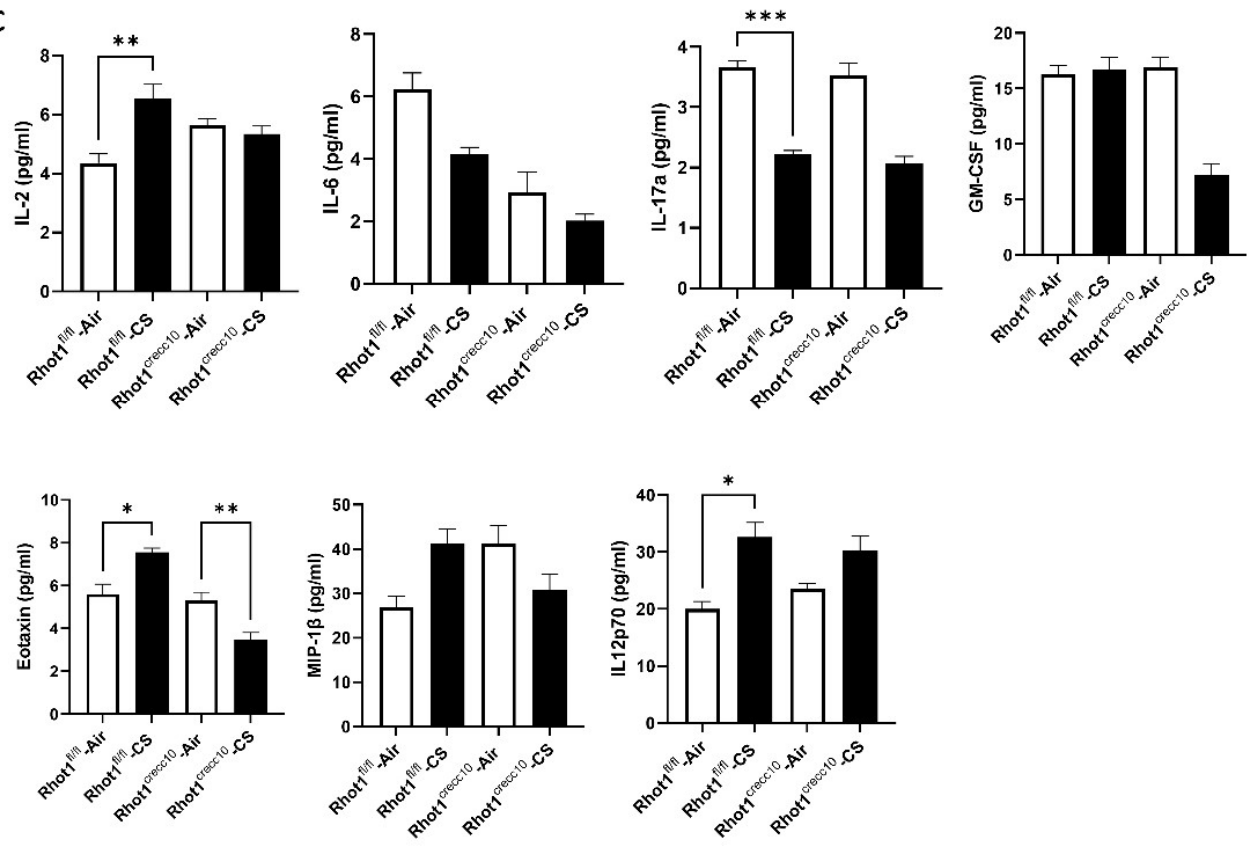

Figure 2 A-C: Differential expression of cytokines in epithelial cell-specific Rhotl deleted and WT mice: Rhot $1^{\mathrm{fl} / \mathrm{fl}}$ (WT) and Rhot $1^{\mathrm{CreCC} 10}$ (Rhot1 flp CreCC10 ${ }^{+/-}$and Rhot1 flp CreCC10 ${ }^{+/+}$) mice were exposed to room air and CS (mainstream) for 3 days (acute exposure). Expression levels of pro-inflammatory and inflammatory mediators in BAL fluid filtered air and CS-exposed mice for 3 days was determined using Bio-Plex Pro 23-plex cytokine assay. Significance compared between corresponding Air and CS groups and all the groups compared with each other irrespective of their exposures. Data are shown as mean $\pm \operatorname{SEM}\left(\mathrm{n}=5\right.$ to 13 per group). $* P<0.05, * * P<0.01,{ }^{* * *} P<$ 0.001 . 
Figure 3

A

Total cell count
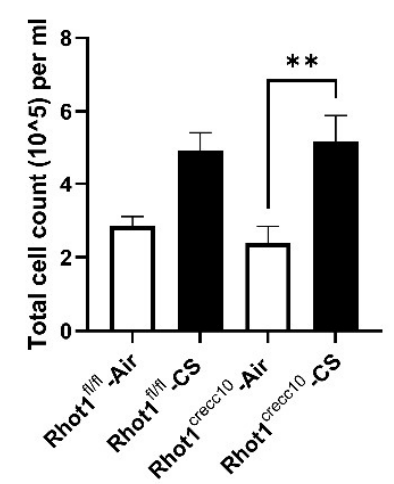

B

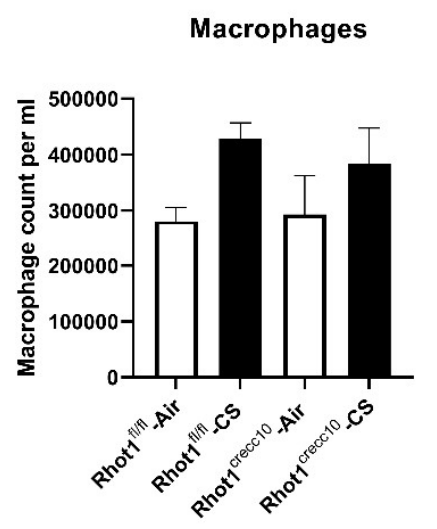

C

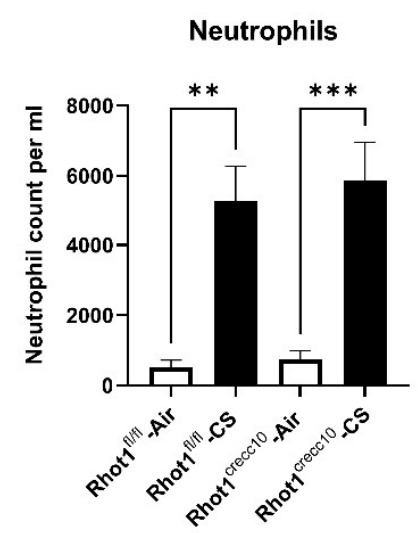

Figure 3: Increased neutrophil influx in $\mathrm{Rhot} 1{ }^{\mathrm{CreCC} 10}$ (Rhot1 Flox CreCC10 $0^{+-}$) mice in response to chronic CS exposure for 4 months. A: The number of total cells in BAL fluid from air and CSexposed mice for 4 months was determined. B: Lavaged macrophage and C: neutrophil numbers were counted in Diff-Quik stained cytospin slides, which were prepared using BAL fluid. Quantification of macrophages and neutrophils expressed as absolute cell count per ml in BAL fluid from air and CS exposed mice. CS exposed Rhot $1{ }^{\mathrm{CreCC} 10}\left(\mathrm{Rhot} 1 \mathrm{CreCC} 10^{+/-}\right)$mice showed significant increase in total cell count and number of neutrophils. Data are shown as mean \pm SEM (n=3 to 5 per group). ${ }^{* *} P<0.01,{ }^{* * *} P<0.001$ significant compared with corresponding air exposed mice 
Figure 4

A
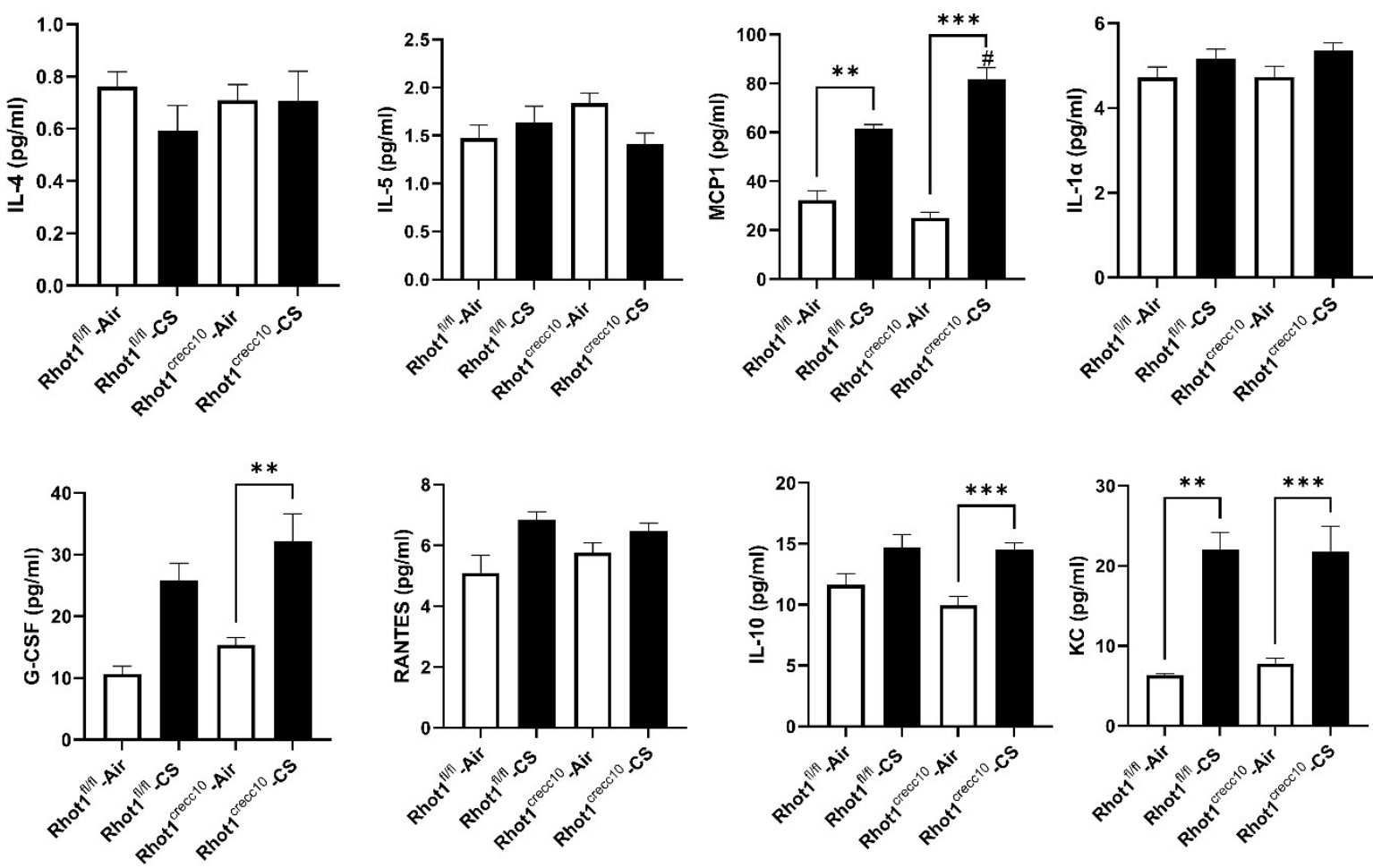
Figure 4

B
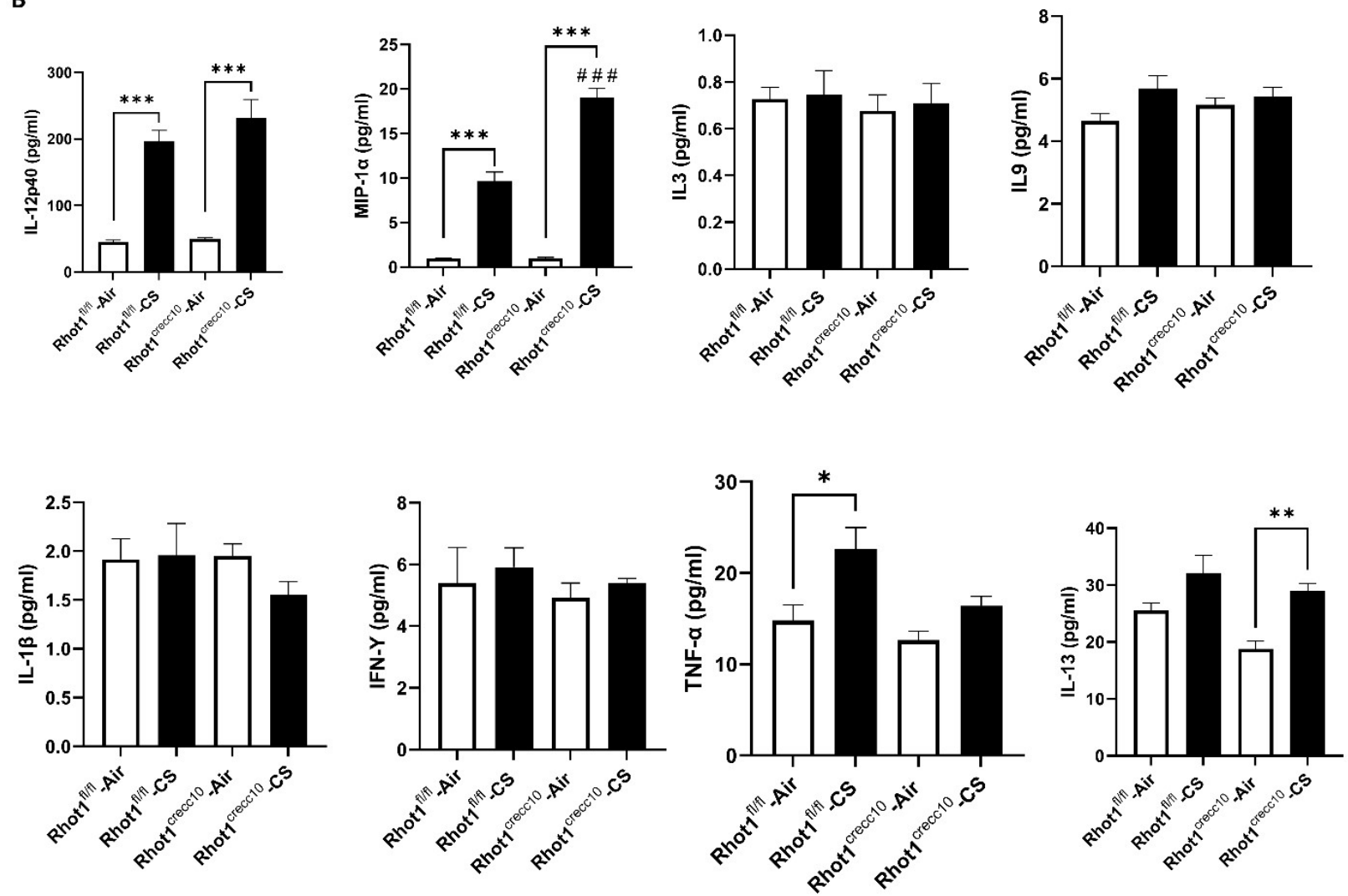
Figure 4

C
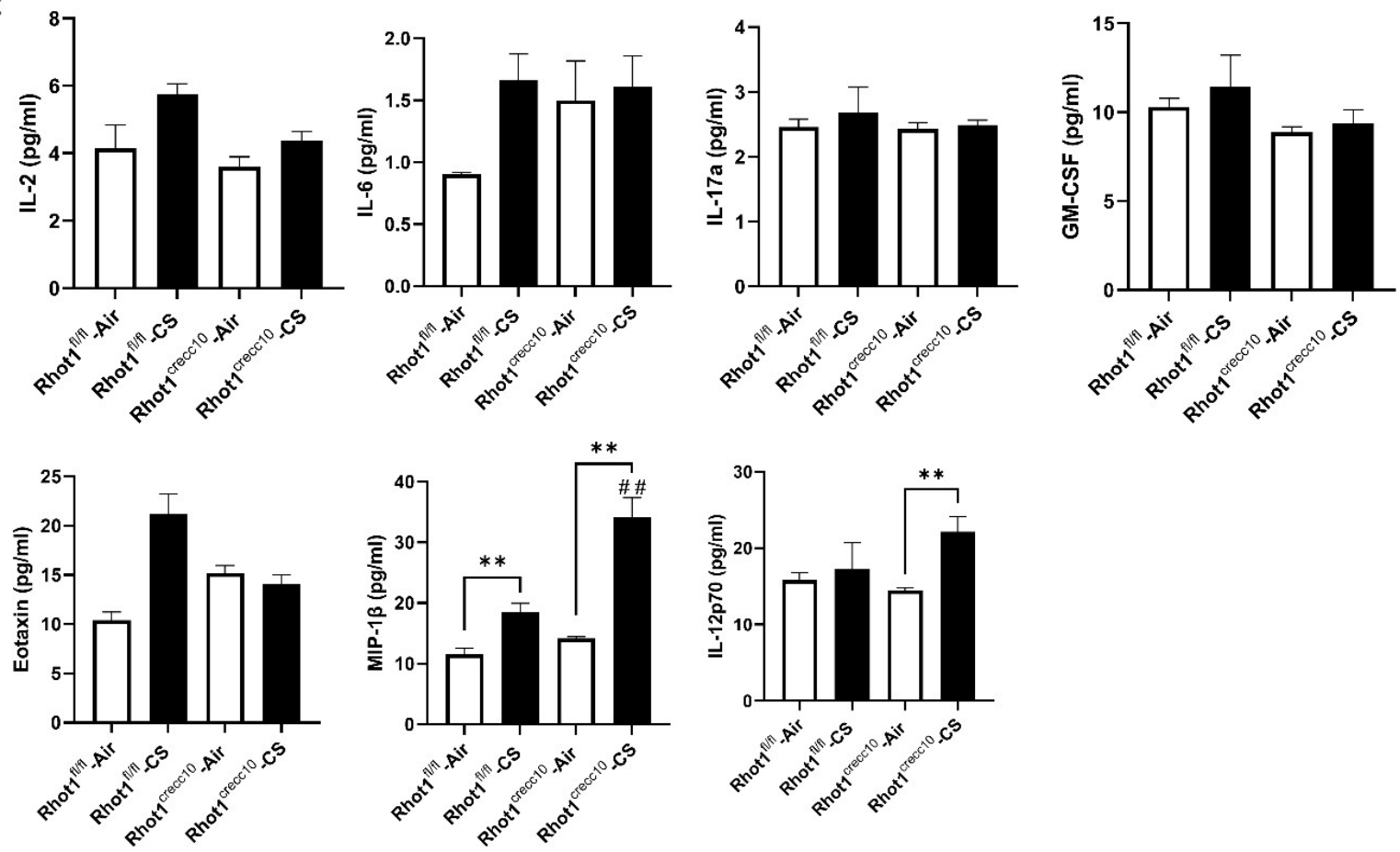

Figure 4A-C: Differential expression of cytokines in epithelial cell-specific Rhot1 deleted and WT mice: Rhot $1^{\mathrm{fl} / \mathrm{fl}}(\mathrm{WT})$ and Rhot $1^{\mathrm{CreCC} 10}\left(\right.$ Rhot1 flp CreCC10 $0^{+/-}$and Rhot1 flp CreCC10 $\left.0^{+/+}\right)$mice were exposed to room air and CS (mainstream) for 4 months (chronic exposure). Expression levels of pro-inflammatory and inflammatory mediators in BAL fluid filtered air and CS-exposed mice for 4 months as determined using Bio-Plex Pro 23-plex cytokine assay. Significance compared between corresponding Air and CS groups and all the groups compared with each other irrespective of their exposures. Data are shown as mean $\pm \operatorname{SEM}\left(\mathrm{n}=5\right.$ to 13 per group). ${ }^{*} P<0.05$, ${ }^{* *} P<0.01,{ }^{* * *} P<0.001$ between groups. ${ }^{\#} P<0.05$, ${ }^{\#} P<0.01$, ${ }^{\# \# \#} P<0.001$ compared to Rhot $^{\mathrm{fl} / \mathrm{fl}}$-CS. 
Figure 5

A Resistance

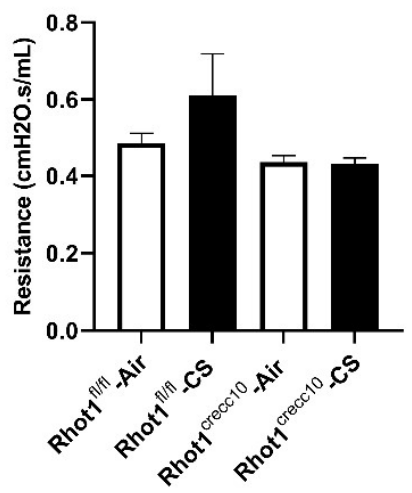

B Elastance
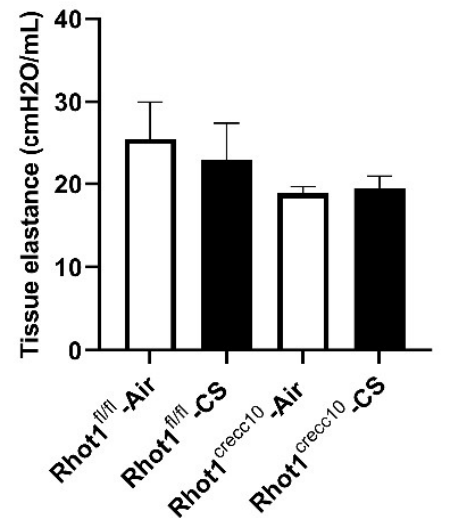

C Compliance
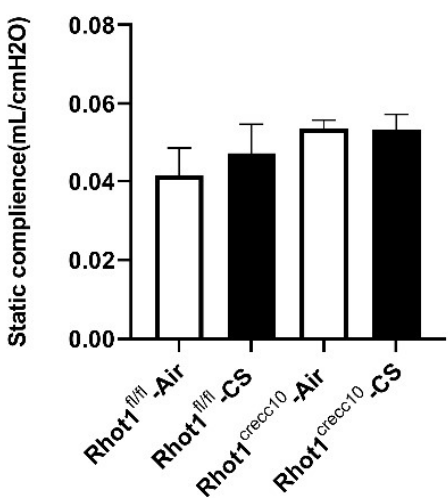

Figure 5: Alterations in lung mechanical properties in $\mathrm{Rhot} 1^{\mathrm{CreCC} 10}\left(\mathrm{Rhot} 1 \mathrm{Flox} \mathrm{CreCC} 10^{+/}\right)$mice in response to $\mathrm{CS}$ exposure for 4 months. A: Tissue resistance B: elastance and C: static compliance were measured by FlexiVent after 4 months of air and CS exposure. Significance compared between corresponding Air and CS groups and all the groups compared with each other irrespective of their exposures, none of the changes found significant. Data are shown as mean \pm SEM ( $\mathrm{n}=3$ to 7 per group). 
Figure 6

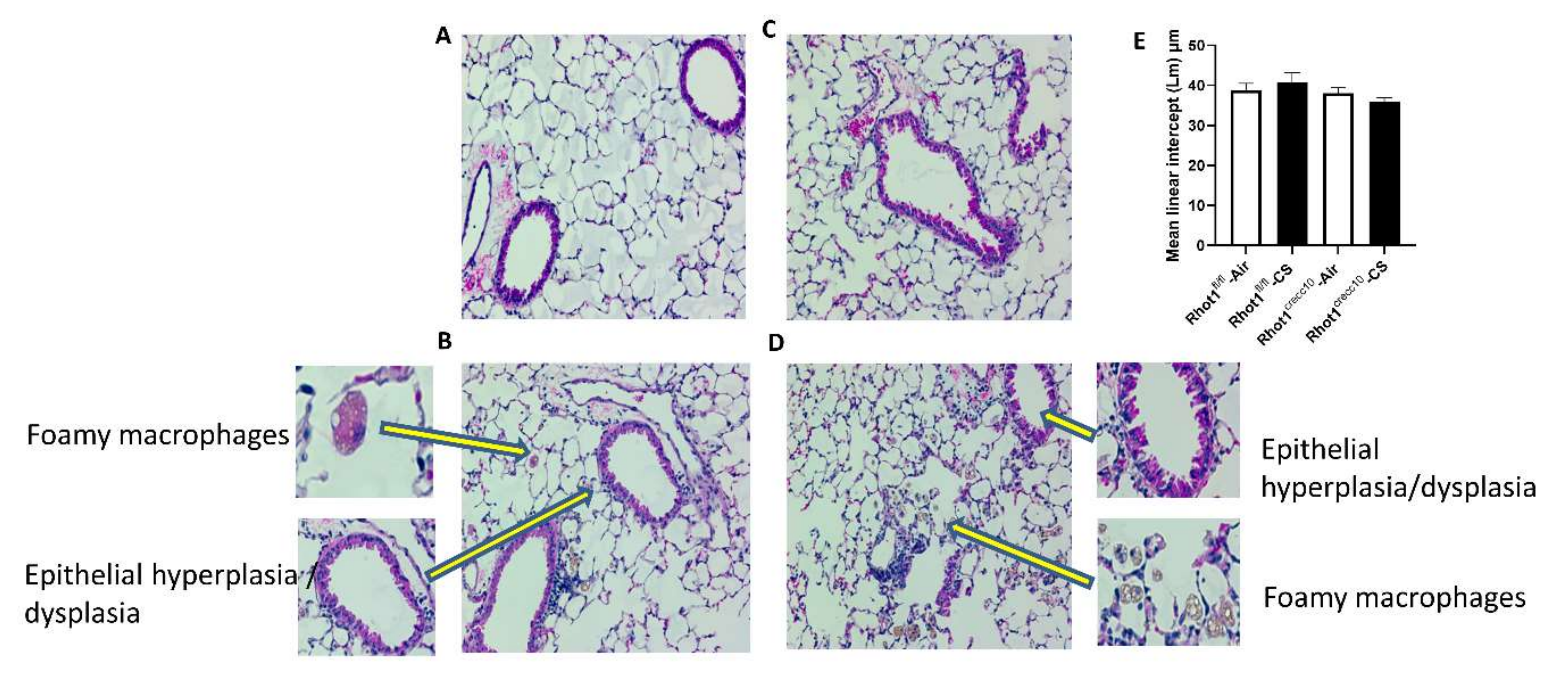

Figure 6: The effect of CS on airspace enlargement and histopathology in lungs of Rhot $1^{\mathrm{CreCC} 10}$ (Rhot1 Flox CreCC10+/- and Rhot1 Flox CreCC10 ${ }^{+/}$) mice exposed to CS for 4 months: areas of interest are magnified and shown in rectangles. The pictures shown are H\&E stained lung sections from air and CS exposed Rhot1 ${ }^{\mathrm{fl} / \mathrm{fl}}(\mathrm{WT})$ and Rhot ${ }^{\mathrm{CreCC} 10}$ (Rhot1 flox CreCC10+/- Rhot1 Flox CreCC10 $0^{+/-}$) mice for 4 months. A Rhot1 $1^{\mathrm{fl} / \mathrm{fl}}$-Air, B Rhot1 $1^{\mathrm{fl} / \mathrm{fl}}$-CS, C Rhot1 ${ }^{\mathrm{CreCC} 10}$-Air, D Rhot $1^{\mathrm{CreCC} 10}-\mathrm{CS}$. Original magnification is $20 \mathrm{X}$. E. Mean Linear Intercept (Lm) was analyzed in H\&E stained- tissue sections. Lung sections from Air and CS-exposed Rhot $1{ }^{\mathrm{CreCC} 10}$ mice did not show significant air space enlargement in comparison with air and CS-exposed Rhot $1^{\mathrm{fl} / \mathrm{fl}}$ (WT) mice. Data are shown as mean \pm SEM ( $n=6$ to 14 per group). However, other histopathological changes such as accumulation of pulmonary macrophages and signs of Epithelial hyperplasia/dysplasia at few sites of the CS exposed mice tissue were observed. The changes are more intensified in $\mathrm{Rhot} 1{ }^{\mathrm{CreCC} 10}$ mice. 
Figure 7

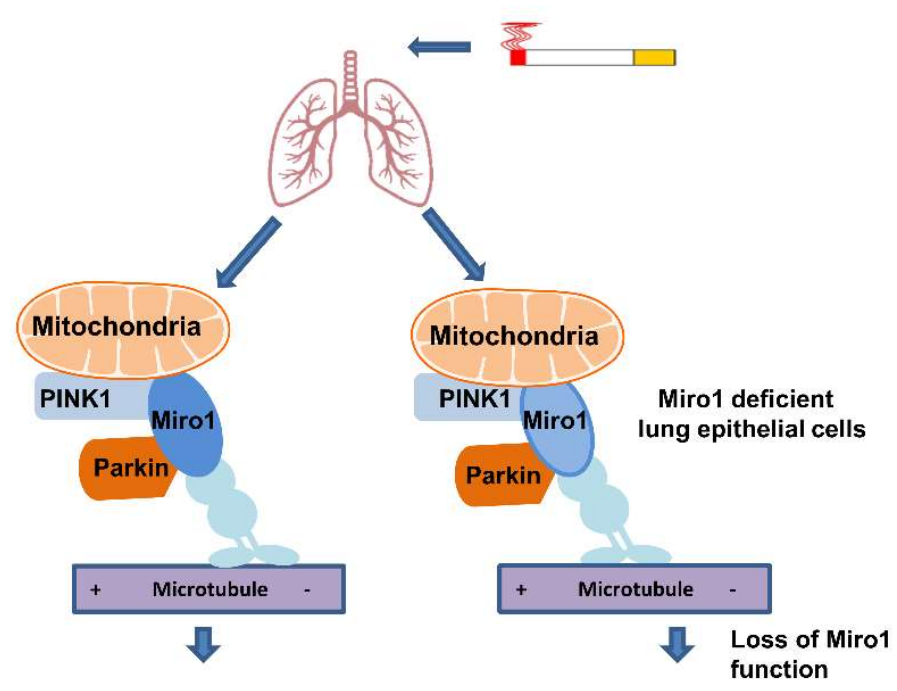

Lung inflammation

Augmented lung inflammation

Figure 7: Role of Miro1 in CS induced lung inflammation 